\title{
Constructal optimisation of conjugate triangular cooling channels with internal heat generation
}

\author{
J.P. Meyer, O.T. Olakoyejo, and T. Bello-Ochende ${ }^{*}$ \\ Department of Mechanical and Aeronautical Engineering, University of Pretoria, Pretoria \\ Private Bag X20, Hatfield 0028, South Africa.
}

\begin{abstract}
This paper presents the development of the three-dimensional flow architecture of conjugate cooling channels in forced convection with internal heat generation within a solid. Two types of cross-section channel geometries were used. The first involved equilateral triangles with three equal legs in length and all three internal angles of $60^{\circ}$. The second was isosceles right triangles with two legs of equal length and internal angles of $90^{\circ}, 45^{\circ}$ and $45^{\circ}$. Both the equilateral triangle and isosceles right triangle are special case of triangle that can easily and uniformly be packed and arranged to form a larger constructs. The configurations were optimised in such a way that the peak temperature of the heat generating solid was minimised subject to the constraint of a fixed global volume of the solid material. The cooling fluid was driven through the channels by the pressure difference across the channel. The degrees of freedom of the channels were aspect ratio, hydraulic diameter and channel to channel spacing ratio. The shape of the channel was allowed to morph to determine the best configuration that gives the lowest thermal resistance. A gradient-based optimisation algorithm was applied in order to search for the best optimal geometric configurations that improve thermal performance by minimising thermal resistance for a wide range of dimensionless pressure difference. The effect of porosities, applied pressure and heat generation rate on the optimal aspect ratio and channel to channel spacing are reported. It was found that there are unique optimal design variables for a given pressure difference. The numerical results that were obtained were in agreement with the theoretical formulation using scale analysis and method of intersection of asymptotes. Results obtained show that the
\end{abstract}

\footnotetext{
* Corresponding author. Tel.: +27 12 4203105; fax: +27 124206632

E-mail address: tunde.bello-ochende@up.ac.za
} 
effects of applied dimensionless pressure drop on minimum thermal resistance were consistent with those obtained in the open literature.

Keywords: Optimisation, Laminar flow, Forced convection, Optimal geometry, Peak temperature, Constructal theory, Thermal resistance, Dynamic-Q

\begin{tabular}{|c|c|c|c|}
\hline \multicolumn{4}{|c|}{ Nomenclature } \\
\hline$A_{c}$ & $\begin{array}{l}\text { Cross sectional area of the } \\
\text { channel, } \mathrm{m}^{2}\end{array}$ & $V$ & Global structure volume, $\mathrm{m}^{3}$ \\
\hline$A_{s}$ & $\begin{array}{l}\text { Cross sectional area of the } \\
\text { structure, } \mathrm{m}^{2}\end{array}$ & $v_{c}$ & Channel volume, $\mathrm{m}^{3}$ \\
\hline$A R_{c}$ & Channels aspect ratio & $v_{e l}$ & Elemental volume, $\mathrm{m}^{3}$ \\
\hline$B e$ & Bejan number & $W$ & Structure width, m \\
\hline$C_{P}$ & $\begin{array}{l}\text { Specific heat at constant } \\
\text { pressure, } \mathrm{J} / \mathrm{kg} \mathrm{K}\end{array}$ & $w$ & Elemental width, m \\
\hline$d_{h}$ & Hydraulic diameter, m & $x, y, z$ & Cartesian coordinates, m \\
\hline$E-T$ & Equilateral triangle & \multicolumn{2}{|c|}{ Greek symbols } \\
\hline$H$ & Structure height, m & $\alpha$ & Thermal diffusivity, $\mathrm{m}^{2} / \mathrm{s}$ \\
\hline$h$ & Elemental height , m & $\beta$ & $\begin{array}{l}\text { Numerical value determined from the } \\
\text { porosity of the channel }\end{array}$ \\
\hline$i$ & Mesh iteration index & $\mu$ & Viscosity, kg/m.s \\
\hline$I-T$ & Isosceles right triangle & $v$ & Kinematics viscosity, $\mathrm{m}^{2} / \mathrm{s}$ \\
\hline$k$ & Thermal conductivity, W/mK & $\rho$ & Density, kg/m³ \\
\hline$L$ & Axial length, m & $\partial$ & Differential \\
\hline$N$ & Number of channels & $\infty$ & Far extreme end, free stream \\
\hline$n$ & Normal & $\phi$ & Porosity \\
\hline$P$ & Pressure, $\mathrm{Pa}$ & $\Delta$ & Difference \\
\hline$P_{o}$ & Poiseuille number & $\nabla$ & Differential operator \\
\hline $\operatorname{Pr}$ & Prandtl number & $\gamma$ & Convergence criterion \\
\hline$\dot{q}^{\prime \prime}$ & heat flux, $\mathrm{W} / \mathrm{m}^{2}$ & \multicolumn{2}{|c|}{ Subscripts } \\
\hline$q_{s}^{\prime \prime \prime}$ & Internal heat generation density, & $f$ & Fluid \\
\hline
\end{tabular}




\begin{tabular}{|llll|}
\hline & W/m ${ }^{3}$ & & \\
$\dot{q}$ & Heat transfer rate, $\mathrm{W}$ & in & Inlet \\
$R$ & Thermal resistance & $\max$ & Maximum, peak \\
$R e$ & Reynolds number & $\min$ & Minimum \\
$s$ & Channel spacing, $\mathrm{m}$ & opt & Optimum \\
$T$ & Temperature, ${ }^{0} \mathrm{C}$ & $s$ & Solid \\
$u$ & Velocity vector, $\mathrm{m} / \mathrm{s}$ & $w$ & Wall \\
\hline
\end{tabular}

\section{Introduction}

Constructal theory and design [1, 2], have emerged as an evolutionary design philosophy for developing flow architectures that offer greater flow access and system performance. This law is stated by Bejan [1, 2] as: For a finite-size system to persist in time (to live), it must evolve in such a way that it provides easier access to the imposed (global) currents that flow through it.

The application of this evolutionary design approach to the discovery of internal heat exchanger started with Bejan and Sciubba [3]. These researchers obtained the design rule for spacing an array of parallel plates to channels so that the heat transfer density of a volume filled with heat-generating components was maximum. The spacing was determined by using the method of asymptotes. This philosophy has been applied to all the facets of flow system design, from biology and physics, to engineering and social organisation [4-10].

In this paper our focus is on the first engineering application of constructal theory, which is the shape and structure for heat transfer and fluid flow [11-14]. The advantage of constructal law in the engineering field is that the flow architecture is not assumed in advance, but is the consequence of allowing the structure to morph [15]. The applications of this theory have been reviewed most recently by Bejan and Lorente [16].

Yilmaz et al. [17] studied the optimum shape and dimensions for convective heat transfer of laminar flow at constant wall temperatures for ducts with parallel plate, circular, square and equilateral triangle geometries. Approximate equations were derived in the form of maximum dimensionless heat flux and optimum dimensionless hydraulic diameter in terms of the duct shape factors and the Prandtl number. 
Also, Bello-Ochende et al. [18] conducted a three-dimensional optimisation of heat sinks and cooling channels with heat flux using scale analysis and the intersection of asymptotes method based on constructal theory to investigate and predict the design and optimisation of the geometric configurations of the cooling channels. Rocha et al. [19] and Biserni et al. [20] applied the theory to optimise the geometry of C-and H-shaped cavities respectively that intrude into a solid conducting wall in order to minimise the thermal resistance between the solid and the cavities. Muzychka [21] studied and analysed the optimisation of microtube heat sinks and heat exchangers for maximum thermal heat transfer by using a multiscale design approach. In this analysis, it was shown that through the use of interstitial microtubes, the maximum heat transfer rate density for an array of circular tubes increased. Page et al. [22], numerically, investigated the thermal behaviour of an assembly of consecutive heat generating cylinders in stationary, rotating and counter-rotating configurations cooled by natural convection with the objective of maximizing the heat transfer density rate by optimising spacing between the consecutive cylinders. Reis et al. [23] optimised the internal configurations of parallel plates and cylindrical channels using contructal theory to understand the morphology of particle agglomeration and the design of air-cleaning devices.

Also, the constructal theory for optimisation of several components and systems and components in engineering applications has been extensively discussed and documented in refs. [24-27].

The recent comment by Meyer [28] on the work Bejan and Lorente [29] on constructal theory shows that the constructal law's application in human life, nature and in all fields of educational design is a wide road to future advances.

This paper focuses on the study of three-dimensional, laminar forced convection cooling in a solid structure by channels with (a) equilateral triangle geometries and (b) isosceles right triangles. It examines the optimisation of a fixed and finite global volume of solid materials with an array of triangular cooling channels, which experience a uniform internal heat generation which will result in a minimal global thermal resistance. The objective is the building of a smaller construct to form a larger construct body that will lead to the minimisation of the global thermal resistance or, inversely, the maximisation of the heat transfer rate density (the total heat transfer rate per unit volume). This is achieved by forcing a coolant to the heated spot in a fast and efficient way so as to reduce the peak temperature at 
any point inside the volume that needs cooling. The optimisation process is carried out numerically under total fixed volume and manufacturing constraints.

This study is an extension of our previous work [27] on the constructal theory for the cylindrical and square configurations with internal heat generation, where we showed that the minimised peak temperature is a function of the geometry and shape. Triangular shapes are considered separately because of the unique nature of the geometry.

\section{Computational model}

The schematic Figure 1a represents the physical configuration of the channels in the solid body with heat generation. The figure shows the triangle as an equilateral triangle but it can also be an isosceles right triangle. The system consists of parallel triangular cooling channels in a solid structure of fixed global volume $V$ with an internal heat generation $q_{s}^{\prime \prime \prime}$. The body is cooled by forcing cooling fluid (water) from the left side into the parallel cooling channels by a fixed pressure difference $\Delta P$ over the channel length $L$. The fluid is assumed to be single phase, steady and Newtonian with constant properties. An elemental volume shown in Figs. 1b and c consisting of isosceles right and equilateral triangular cooling channels respectively and the surrounding solid used for analysis because of the assumption of the symmetrical heat distribution inside the structure. The heat transfer in the elemental volume is a conjugate problem, which combines heat conduction in the solid and the convection in the flowing fluid.

\section{Design variables for isosceles right triangle and equilateral triangle}

In Fig. 1b, for isosceles right triangular channel shape, an elemental volume, $v_{e l}$, constraint is considered to be composed of an elemental cooling channel of hydraulic diameter, $d_{h}$, and the surrounding solid of thickness $s_{1}$ and $s_{2}$ defined as:

$$
\begin{aligned}
& w=h, \quad v_{e l}=w^{2} L, \quad h_{c}=\frac{w_{c}}{2}, \\
& d_{h}=\frac{w_{c}^{2}}{w_{c}+\sqrt{2} w_{c}}, \quad v_{c}=\frac{w_{c}^{2}}{4} L
\end{aligned}
$$

However, the void fraction or porosity of the unit structure can be defined as:

$$
\phi=\frac{v_{c}}{v_{e l}}=\frac{w_{c}}{4 w}
$$

Again, in Fig. 1c, equilateral triangular channel shape, the design variables are defined as: 


$$
\begin{aligned}
& w=h, \quad v_{e l}=w^{2} L, \quad h_{c}=\frac{\sqrt{3}}{2} w_{c}, \\
& d_{h}=\frac{1}{\sqrt{3}} w_{c}, \quad v_{c}=\frac{\sqrt{3}}{4} w_{c}{ }^{2} L
\end{aligned}
$$

The void fraction or porosity of the unit structure can be defined as:

$$
\phi=\frac{v_{c}}{v_{e l}}=\frac{\sqrt{3}}{4} \frac{w_{c}}{w}
$$

For a fixed length of the channel, we have

$$
A_{s}=H W
$$

Therefore, the total number of channels in the structure arrangement for the two configurations can be defined as:

$$
N=\frac{H W}{h w}=\frac{H W}{\left(h_{c}+s_{2}\right)\left(w_{C}+s_{1}\right)}
$$

Some other assumptions imposed on the two triangular configurations are: the solid structure top and bottom boundaries of the domain correspond to periodic boundary conditions, the left and right side of the solid surfaces were taken as symmetry boundary conditions. All the outside walls were taken as a plane of symmetry of the solid structure and were modelled as adiabatic as shown in Figs. 1b and c.

The objective is to find the Aspect ratio $\mathrm{AR}_{c}$, hydraulic diameter, $d_{h}$, and the channel spacing, which offer minimum resistance to heat and flow.

The temperature distribution in the model was determined by solving the equation for the conservation of mass, momentum and energy numerically.

The governing differential equations used for the fluid flow and heat transfer analysis in the unit volume of the structure are:

$$
\begin{aligned}
& \nabla \cdot \vec{u}=0 \\
& \rho(\vec{u} \cdot \nabla \vec{u})=-\nabla p+\mu \nabla^{2} \vec{u} \\
& \rho_{f} C_{P f}(\vec{u} \cdot \nabla T)=k_{f} \nabla^{2} T
\end{aligned}
$$

The energy equation for a solid with internal heat generation is given as:

$$
k_{s} \nabla^{2} T+q_{s}^{\prime \prime \prime}=0
$$

The continuity of the heat flux at the interface between the solid and the liquid is given as:

$$
k_{s} \frac{\partial T}{\partial n}\left|=k_{f} \frac{\partial T}{\partial n}\right|
$$


A no slip boundary condition is specified at the wall of the channel, $\vec{u}=0$, at the inlet $(x=$ $0), u_{x}=u_{y}=0, T=T_{\text {in }}$ and

$$
P_{\text {in }}=\frac{B e \alpha u}{L^{2}}+P_{o u t}
$$

where $B e$, is the dimensionless pressure difference defined in Refs [30, 31]

At the outlet ( $x=L$ ), a zero normal stress is specified, and the pressure is $P_{\text {out }}=1$ atm

At the solid boundaries,

$\nabla T=0$

The measure of performance is the minimum global thermal resistance, which could be expressed in a dimensionless form as:

$$
R_{\min }=\frac{k_{f}\left(T_{\max }-T_{i n}\right)_{\min }}{q_{S}^{\prime \prime \prime} L^{2}}
$$

and it is a function of the optimised design variables and the peak temperature.

$$
R_{\min }=f\left(A R_{c}, d_{h}, s_{1}, s_{2}, \phi,\left(T_{\max }\right)_{\min }\right)
$$

The inverse of $R$ is the global thermal conductance

\section{Numerical procedure and grid analysis}

The simulation work began by fixing the length of the channel, applied pressure difference, porosity, Internal heat generation and material properties and we kept varying the values of the aspect ratio and hydraulic diameter of the channel in order to identify the best (optimal) internal configuration that minimised the peak temperature. The numerical solution of the continuity, momentum and energy Eqs. (7) - (10) along with the boundary conditions (11) - (13) was obtained by using a three-dimensional commercial package FLUENT ${ }^{\mathrm{TM}}$ [32], which employs a finite volume method. The details of the method were explained by Patankar [33]. FLUENT ${ }^{\mathrm{TM}}$ was coupled with a geometry and mesh generation package GAMBIT [34] using MATLAB [35] to allow the automation and running of the simulation process. After the simulation had converged, an output file was obtained containing all the necessary simulation data and results for the post-processing and analysis. The computational domain was discretised using hexahedral/wedge elements. A second-order upwind scheme was used to discretise the combined convection and diffusion terms in the momentum and energy equations. The SIMPLE algorithm was then employed to solve the coupled pressure-velocity fields of the transport equations. The solution is assumed to have converged when the 
normalised residuals of the mass and momentum equations fall below $10^{-6}$ and while the residual convergence of energy equation was set to less than $10^{-10}$. The number of grid cells used for the simulations varied for different elemental volume and porosities. However, grid independence tests for several mesh refinements were carried out to ensure the accuracy of the numerical results. The convergence criterion for the overall thermal resistance as the quantity monitored is:

$$
\gamma=\frac{\left|\left(T_{\max }\right)_{i}-\left(T_{\text {max }}\right)_{t-1}\right|}{\left|\left(T_{\text {max }}\right)_{i}\right|} \leq 0.01
$$

where $i$ is the mesh iteration index. The mesh is more refined as $i$ increases. The $i-1$ mesh is selected as a converged mesh when criterion (16) is satisfied.

To ensure accurate results, several grid independence tests were conducted until a mesh size with negligible changes in peak temperature was obtained.

Tables 1 and 2 show the grid independence test for isosceles right triangular and equilateral triangular configurations with $v_{e l}=0.4 \mathrm{~mm}^{3} \phi=0.2$, and $L=10 \mathrm{~mm}$ for $\Delta P=50 \mathrm{kPa}$. Also, computational cell densities of 32 526, 59 000, 76728 and 118750 were used for the grid independence test for isosceles right triangular configuration. It was observed that almost identical results were predicted when 59000 and 76728 cells were used. Therefore, any increase in the cell density beyond 59000 had a negligible effect on the numerical result.

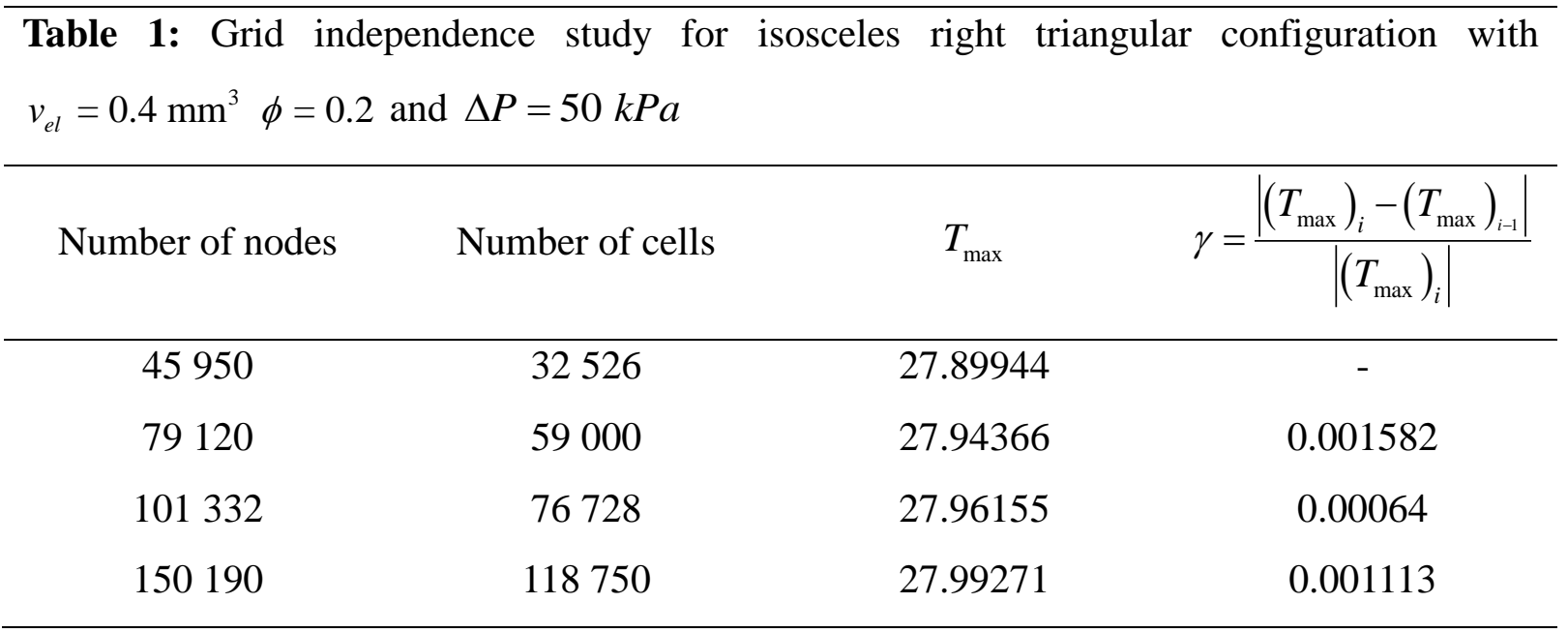

Again, computational cell densities of 19 314, 33 360, 63500 and 77284 were used for the grid independence test for the equilateral triangular configuration. It was observed that almost identical results were predicted when 63500 and 77284 cells were used. Therefore, a further 
increase in the cell density beyond 63500 would have a negligible effect on the numerical result.

Table 2: Grid independence study for equilateral triangular configuration with $v_{e l}=0.4 \mathrm{~mm}^{3}$ $\phi=0.2$ and $\Delta P=50 \mathrm{kPa}$

\begin{tabular}{cccc}
\hline Number of nodes & Number of cells & $T_{\max }$ & $\gamma=\frac{\left|\left(T_{\max }\right)_{i}-\left(T_{\max }\right)_{i-1}\right|}{\left|\left(T_{\max }\right)_{i}\right|}$ \\
\hline 28034 & 19314 & 27.81091 & - \\
46786 & 33360 & 27.8580 & 0.00169 \\
83629 & 63500 & 27.90048 & 0.001523 \\
101889 & 77284 & 27.91846 & 0.000644 \\
\hline
\end{tabular}

\section{Numerical results}

In this section, we present results for the case when the elemental volume was in the range of $0.025 \mathrm{~mm}^{3} \leq v_{e l} \leq 5 \mathrm{~mm}^{3}$ and the porosities ranged between $\phi=0.2$ with a fixed length of $L=10 \mathrm{~mm}$ and fixed applied dimensionless pressure differences of $\Delta P=50 \mathrm{kPa}$. The thermal conductivity of the solid structure (silicon) is $148 \mathrm{~W} / \mathrm{m} . \mathrm{K}$, and the internal heat generation within the solid was taken to be fixed at $100 \mathrm{~W} / \mathrm{cm}^{3}$. The thermophysical properties of water [36] used in this study were based on water at $300 \mathrm{~K}$ and the inlet water temperature was fixed at this temperature.

Figure 2 shows the existence of an optimal hydraulic diameter and optimum elemental volume of the structure that minimised the peak temperature at any point in the channel for the two types of triangular configurations studied. The abbreviation (E-T) is used for equilateral triangle and (I-T) is used for isosceles right triangle. Fig. 2a shows the peak temperature as a function of the channel dimensionless hydraulic diameter for prescribed pressure difference. It shows that there exists an optimum channel hydraulic diameter, which lies in the range $0.005 \leq d_{h} / L \leq 0.02$ and minimises the peak temperature. Also, the elemental volume of the structure has a strong effect on the peak temperature as shown in Fig. 2b. The minimum peak temperature is achieved when the optimal elemental volume of the structure minimizes the peak temperature and this lies in the range of $0.2 \mathrm{~mm}^{3} \leq v_{e l} \leq 2.5 \mathrm{~mm}^{3}$. Any further increase or decrease in the design variable beyond the optimal values indicates that the working fluid is not properly engaged in the cooling process. This is detrimental to the global 
performance of the system. The results also, show that the optimal arrangement of the elemental volume for the entire structure at this fixed pressure difference should be very small in order to achieve better cooling.

\section{Mathematical optimisation}

In this section, we introduce an optimisation algorithm that will search and identify the optimal design variables at which the system will perform at an optimum. A numerical algorithm, Dynamic-Q [37] was employed and incorporated into the finite volume solver and grid (geometry and mesh) generation package by using MATLAB. The Dynamic-Q is a multidimensional and robust gradient-based optimisation algorithm, which does not require an explicit line search. The technique involves the application of a dynamic trajectory LFOPC optimisation algorithm to successive quadratic approximations of the actual problem [38].

The algorithm is also specifically designed to handle constrained problems where the objective and constraint functions are expensive to evaluate. The details of the Dynamic-Q and applications can be found in Refs [14, 27, 37-39].

\section{Optimisation problem}

\section{1. $\quad$ Design variables constraints}

The constraint ranges for the triangular configuration optimisation were:

$$
\begin{aligned}
& 0.025 \mathrm{~mm}^{3} \leq v_{e l} \leq 5 \mathrm{~mm}^{3}, \quad 0.1 \leq \phi \leq 0.2, \quad h=w, \quad 0 \leq \mathrm{w}_{C} \leq w, \\
& 0 \leq h_{C} \leq h, \quad 0 \leq d_{h} \leq w, \quad 0 \leq s_{1} \leq w, \quad 0 \leq s_{2} \leq w
\end{aligned}
$$

The optimisation process was repeated for applied dimensionless pressure differences $(\mathrm{Be})$ that correspond to $\triangle P=5 \mathrm{kPa}$ to $\triangle P=50 \mathrm{kPa}$.

Figure 3a shows the minimised dimensionless global thermal resistance as a function of dimensionless pressure difference at different porosities for the two triangular configurations. The results show that the minimised dimensionless global thermal resistance monotonically decreases as the dimensionless pressure difference increases. Also Figs. 3b and c show the optimal behaviours of the geometry with respect to applied dimensionless pressure difference (or Bejan number) at different porosities for the two triangular configurations. Fig. 3b shows that the optimal hydraulic diameter decreases as the dimensionless pressure differences increase and there exists a unique optimal geometry for each of the applied dimensionless pressure differences for the configurations. These trends of results are also in good agreement with previous work [27, 39]. 
Figure 3c shows the optimal channel spacing ratio $\left(\mathrm{s}_{1} / \mathrm{s}_{2}\right)$ remains unchanged and insensitive to the performance of the system whereas regardless of the dimensionless pressure difference number for the two triangular configurations. This constant value could be described as allowable spacing due to manufacturing constraints. This implies that the closer the channels are to one another, the better the effective cooling ability of the global system will be.

\section{Method of intersection of asymptotes}

This section investigated further the numerical solution of the optimisation of flow and heat transfer with the analytical solution. The theoretical analysis for the configurations followed the application of the intersection of asymptotes method and scale analysis $[19,27,40]$ to provide the existence of an optimum geometry that minimised the peak temperature and global thermal resistance. The method of intersection of asymptotes outlined by Olakoyejo et al. [27] was used to determine the optimal geometric shape. The objective was to provide the relationship between the global objective function in terms of global thermal resistance, $R$, and the varying hydraulic diameter, $d_{h}$, in the two extremes at $d_{h} \rightarrow 0$ and $d_{h} \rightarrow \infty$. The optimal geometry value, $d_{h_{\text {opt }}}$, that corresponds to, $R_{\min }$, is located approximately where the two asymptotes intercept. The following assumptions were made throughout the analysis: inlet temperature and the pressure difference, $\Delta P$, driving the pump are fixed with a uniform flow distribution in all the channels, laminar flow, constant cross-sectional area of the channels, negligible inlet and exit plenum losses, negligible axial conduction. An elemental volume is treated because of the symmetry of the heat distribution. The details of the analytical procedure is completely analogous to what is presented by Olakoyejo et al. [27], Therefore, the dimensionless thermal resistance, $R$, behaviour in the extreme limit of a small triangular channel is given as:

$$
R=\left[\frac{{ }_{f}\left(T_{\max }-T_{i n}\right)}{q^{\prime \prime \prime} L^{2}}\right] \cong 4 P_{o_{d_{h}}} \beta\left(\frac{d_{h}}{L}\right)^{-2} B e^{-1}
$$

Where $\beta$ is the numerical value determined from the porosity of the channel and is defined in re[27] as: 
$\beta=\left(\frac{1-\phi}{\phi}\right)$

From Eq. (18) for a smaller channel $d_{h} \ll L$, the thermal resistance is inversely proportional to $d_{h}{ }^{2}$. Keeping $\beta$ (which is a function of porosity) constant, it shows that the global thermal resistance increases as the hydraulic diameter decreases.

In the opposite extreme limit (large channel), the dimensionless global thermal resistance, $R$, is defined in terms of dimensionless pressure difference as:

$$
R=\left[\frac{k_{f}\left(T_{\max }-T_{i n}\right)}{q^{\prime \prime \prime} L^{2}}\right] \cong 0.7643 \beta\left(\frac{d_{h}}{L}\right)^{2 / 3} B e^{-1 / 3}
$$

From Eq. (20), for a larger channel, the global thermal resistance is directly proportional to $d_{h}{ }^{2 / 3}$. Keeping $\beta$ (which is a function of porosity) constant, confirms that as the hydraulic diameter becomes lager, the global thermal resistance increases.

The geometric optimisation in terms of channel could be achieved by combining Eqs. (18) and (20) using the intersection of asymptotes method and the optimal dimension can be generally approximated for the configuration as hydraulic diameter where the two extreme curves intersect. The intersection result is:

$\left(\frac{d_{h}}{L}\right)_{o p t} \approx 1.8602 P_{o_{h}}^{3 / 8} \mathrm{Be}^{-1 / 4}$

where $d_{h_{\text {opt }}}$ is the optimal hydraulic diameter

for an isosceles right triangle channel with hydraulic diameter $d_{h}, P_{o_{d_{h}}}=6.577$, and hence Eq.

(21) reduces to:

$\left(\frac{d_{h}}{L}\right)_{o p t} \approx 3.7698 B e^{-1 / 4}$

for an equilateral triangle channel with hydraulic diameter $d_{h}, P_{o_{d_{h}}}=6.625$, and hence Eq.

(21) reduces to:

$\left(\frac{d_{h}}{L}\right)_{\text {opt }} \approx 3.7801 B e^{-1 / 4}$

The minimum dimensionless global thermal resistance can be obtained for an elemental volume for the configurations that correspond to the optimal geometries by substituting Eq. (21) into Eq. (18) as: 
$R_{\min }=1.156 \beta P_{d_{h}}{ }^{1 / 4} B e^{-1 / 2}$

Eq. (24) shows that the thermal resistance decreases monotonically as $B e$ increases for a fixed porosity. The minimised dimensionless global thermal resistance of a isosceles right triangle channel with $P_{o_{h}}=6.577$ is:

$R_{\min }=1.8513 \beta B e^{-1 / 2}$

and the minimised dimensionless global thermal resistance of a an equilateral triangular channel with $P_{o_{h}}=6.625$ is:

$R_{\min }=1.8546 \beta B e^{-1 / 2}$

\section{Correlations of the theoretical method and numerical optimisation}

The analytical results of Eqs. (21) to (26) were used to validate the numerical solutions. The numerical and approximate solutions based on scale analysis at optimal geometry dimensions are in good agreement and the solutions have similar trends as shown in Fig. 4.

Fig. 4a shows the minimised dimensionless global thermal resistance as a function of the dimensionless pressure difference at optimised design variables for the two triangular configurations. The analytical and the numerical results show that the minimised global thermal resistance group decreases as the dimensionless pressure difference increases. Although the analytical results are lower than numerical results, the theoretical and numerical values agree within a factor of 1.5 for the worst case. However, this deviations, which are attributed to simplifying assumptions made in the formulation of the theoretical solution. These results are also in agreement with past research work [18, 27].

Also Fig. $4 \mathrm{~b}$ shows the effect of the dimensionless pressure difference on the optimised dimensionless hydraulic diameter for the triangular configurations. The curves show that the optimised dimensionless hydraulic diameter decreases as the applied dimensionless pressure difference for different porosities. The results also, show that unique optimal design geometry exists for each applied dimensionless pressure difference and porosity for each of the configuration studied. 


\section{Conclusion}

This paper studied the numerical optimisation of geometric structures of cooling volumes with internal heat generation isosceles right triangular channel and equilateral triangular channel cross-sectional shapes based on constructal theory.

The effects of different geometrical parameters such as the hydraulic diameters, and channel spacing and elemental volume were comprehensively studied. The numerical results obtained were in agreement with the theoretical formulation using scale analysis and method of intersection of asymptotes. Based on the simulated results and analytical solution, the results showed that there was an optimal geometry for the two channel configurations considered which minimises the peak temperature and hence thermal resistance.

Also, the numerical result shows that the dimensionless global thermal resistance for the two triangular configurations were almost the same, though that of isosceles right triangular was slightly lower than that of equilateral triangular. The numerical analysis also showed that the optimised geometry and minimised thermal resistance were functions of the dimensionless pressure difference for different porosities. This shows the existence of unique optimal design variables (hydraulic diamters) for a given applied dimensionless pressure number for each configuration. The results also show that the minimised peak temperature decreases as the porosity increases.

The optimal channel spacing ratio remains unchanged and insensitive to the performance of the system whereas regardless of the dimensionless pressure difference number for the two triangular configurations. This constant value could be described as allowable spacing due to manufacturing constraints. This implies that the closer the channels are to one another, the better the effective cooling ability of the global system.

The use of the optimisation algorithm coupled to the CFD package made the numerical results to be more robust with respect to the selection of optima structures' geometries, internal configurations of the flow channels and dimensionless pressure difference.

\section{Acknowledgements}

The funding obtained from NRF, TESP, Stellenbosch University / University of Pretoria, SANERI/SANEDI, CSIR, EEDSM Hub and NAC is acknowledged and duly appreciated 


\section{References}

[1] A. Bejan, Advanced Engineering Thermodynamics, 2nd ed., Wiley, New York, 1997.

[2] A. Bejan, Shape and Structure from Engineering to Nature, Cambridge University Press, Cambridge, UK, 2000

[3] A. Bejan, E. Sciubba, The Optimal Spacing of Parallel Plates Cooled By Forced Convection, International Journal of Heat and Mass Transfer 35 (1992) 3259-3264.

[4] T. Bello-Ochende, J.P. Meyer, J. Dirker, Three-dimensional multi-scale plate assembly for maximum heat transfer rate density, International Journal of Heat and Mass Transfer, 53 (2010) 586-593.

[5] A. Bejan, V. Badescu, A. De Vos, Constructal theory of economics, Applied . Energy. 67 (2000) 37-60.

[6] A. Bejan, Optimal internal structure of volumes cooled by single phase forced and natural convection, J. Electron. Packaging 125 (2003) 200-207.

[7] Bejan A. Two hierarchies in science: the free flow of ideas and the academy. Int $\mathrm{J}$ Design Nature Ecodyn 4 (2009) 386-94.

[8] O. N. Sara, S. Yapici, M. Yilmaz, T. Pekdemir, Second Law Analysis of Rectangular Channels with Square Pin-fins. Int. Comm. Heat and Mass Transfer 28 (2001) 617630.

[9] A. H. Reis, A.F Miguel, M. Aydin. Constructal theory of flow architecture of the lungs. Med Phys 31 (2004) 1135-40.

[10] X.-Q. Wang, A.S. Mujumdar, C. Yap, Effect of orientation for phase change material (PCM) - based heat sinks for transient thermal management of electronic components, Int. Comm. Heat and Mass Transfer 34 (2007) 801-808.

[11] T. Bello-Ochende, J.P. Meyer, A. Bejan, Constructal ducts with wrinkled entrances, Int. J. Heat Mass Transfer 52 (2009) 3628-3633.

[12] L.O.A. Rocha, E. Lorenzini, C. Biserni, Geometric optimization of shapes on the basis of Bejan’s Constructal theory, Int. Comm. Heat and Mass Transfer 32 (2005) 1281-1288.

[13] M.R. Salimpour, M. Sharifhasan, E. Shirani, 2010, Constructal optimization of the geometry of an array of micro-channels, Int. Comm. Heat and Mass Transfer 38 (2010) 93-99. 
[14] O.T. Olakoyejo, T. Bello-Ochende, J.P. Meyer, Mathematical optimisation of laminar forced convection heat transfer through a vascularised solid with square channels, Int. J. Heat Mass Transfer 55 (2012) 2402-2411.

[15] A.H. Reis, Constructal Theory - Complex flow structures in engineering and in Nature in III Conferência Nacional em Mecânica de Fluidos, Termodinâmica e Energia (MEFTE - BRAGANÇA 09), (2009) 1- 17

[16] A. Bejan, S. Lorente, Design with Constructal Theory, Hoboken Wiley, 2008.

[17] A. Yilmaz, O. Buyukalaca, T. Yilmaz, Optimum shape and dimensions of ducts for convective heat transfer in laminar flow at constant wall temperature, Int. J. Heat Mass Transfer 43 (2000) 767-775.

[18] T. Bello-Ochende, L. Liebenberg, J. P. Meyer, Constructal cooling Channels for micro-channel heat sinks, Int. J. Heat Mass Transfer 50 (21-22) 4141-4150.

[19] L.A.O Rocha, E. Lorenzini, C. Biserni, Y. Cho, Constructal design of a cavity cooled by convection, Int. J. Des. Ecodyn 5 (2010) 212-220.

[20] C. Biserni, L.A.O. Rocha G. Stanescu, and E. Lorenzini, Constructal H-shaped cavities according to Bejan’s theory Int. J. Heat Mass Transfer 50 (2007) 2132-2138.

[21] Y. S. Muzychka, Constructal multi-scale design of compact micro-tube heat sinand heat exchangers, Int J. Thermal Sciences 46 (2007) 245-252.

[22] L.G. Page, T. Bello-Ochende, J.P. Meyer, Maximum heat transfer density rate enhancement from cylinders rotating in natural convection, Int. Comm. Heat and Mass Transfer 38 (2011) 1354-1359

[23] A.H. Reis, A.F. Miguel, A. Bejan, Constructal Theory of particle agglomeration of design of air-cleaning devices , J. Phys. D: Appl. Phys. 39 (2006) 3086-3096.

[24] T. Bello-Ochende, J.P. Meyer, A. Bejan, Constructal multi-scale pin fins, International Journal of Heat and Mass Transfer 53 (2010) 2773-2779.

[25] G. Lorenzini, C. Biserni, L.A.O. Rocha, Geometric optimization of isothermal cavities according to Bejan’s theory. Int. J. Heat Mass Transfer 54 (2011) 3868-3873.

[26] Y. Kim, S. Lorente, A. Bejan, Constructal Multi-tube Configuration for Natural and Forced convection in cross-flow, Int. J. Heat Mass Transfer 53 (2010) 5121-5128.

[27] O.T. Olakoyejo, T. Bello-Ochende, J.P. Meyer, Constructal conjugate cooling channels with internal heat generation, Int. J. Heat Mass Transfer 55 (2012) 43854396. 
[28] J.P. Meyer, Constructal Law in Technology, Thermofluid and Energy Systems, and in Design Education, Phys of life Rev 8 (2011) 247-248.

[29] A. Bejan, S. Lorente, The constructal law and the evolution of design in nature, Phys of life Rev 8 (2011) 209-240.

[30] S. Bhattacharjee, W.L. Grosshandler, The formation of wall jet near a high temperature wall under microgravity environment, ASME HTD, 96, (1998) 711-716.

[31] S. Petrescu, Comments on the optimal spacing of parallel plates cooled by forced convection, Int. J. Heat Mass Transfer 37 (994) 1283.

[32] Fluent Inc., Fluent Version 6 Manuals, Centerra Resource Park, 10 Cavendish Court, Lebanon, New Hampshire, USA, 2001 (www.fluent.com).

[33] S. V. Patankar, Numerical Heat Transfer and Fluid flow”, Hemisphere, New York. 1980.

[34] Fluent Inc., Gambit Version 6 Manuals, Centerra Resource Park, 10 Cavendish Court, Lebanon, New Hampshire, USA, 2001 (www.fluent.com).

[35] The MathWorks, Inc., MATLAB \& Simulink Release Notes for R2008a, 3 Apple Hill Drive, Natick, MA, 2008 (www.mathworks.com).

[36] F.M. White, Viscous Fluid Flow, 2nd Edition, McGraw-Hill International Editions, Singapore, 1991.

[37] J.A. Snyman, A.M. Hay, The DYNAMIC-Q Optimization Method: An alternative to SQP? Computer and Mathematics with Applications 44 (2002) 1589-1598.

[38] J.A. Snyman, N. Stander, W.J. Roux, dynamic penalty function method for the solution of structural optimization problems, Appl. Math. Model. 18 (1994) 453-460.

[39] T. Bello-Ochende, J.P. Meyer, F.U. Ighalo, Combined Numerical Optimization and Constructal Theory for the Design of Microchannel Heat Sinks, Numerical Heat Transfer, Part A, 58 (2010) 882-899.

[40] A. Bejan, Convection Heat Transfer, third ed., Wiley, Hoboken, 2004. 


\section{List of Figures}

Figure 1. (a) Three-dimensional triangular channels across a solid body with internal heat generation and forced flow and (b) The boundary conditions of the three dimensional computational domain of equilateral triangular cooling channel, (c) An isosceles right with the same boundary conditions as in (1b)

Figure 2. Effect of (a) optimised hydraulic diameter $d_{h}$, on the peak temperature (b) optimised elemental volume $v_{e l}$, on the peak temperature

Figure 3. Effect of dimensionless pressure difference on (a) the minimised dimensionless global thermal resistance, (b) optimised hydraulic diameter, (c) optimised channel spacing ratio

Figure 4. Correlation of numerical and analytical solutions for (a) the minimised global thermal resistance and (b) the optimised hydraulic diameter 


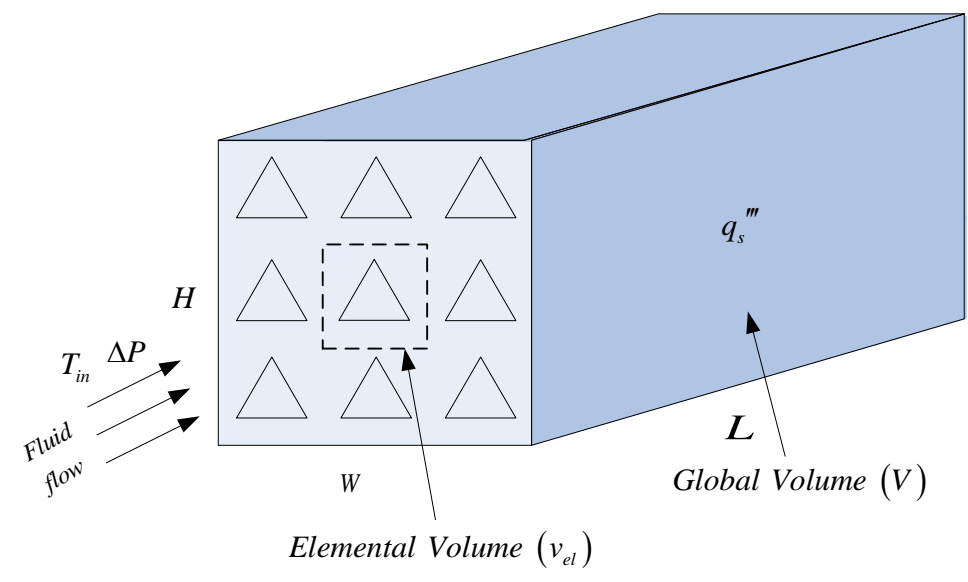

(a)

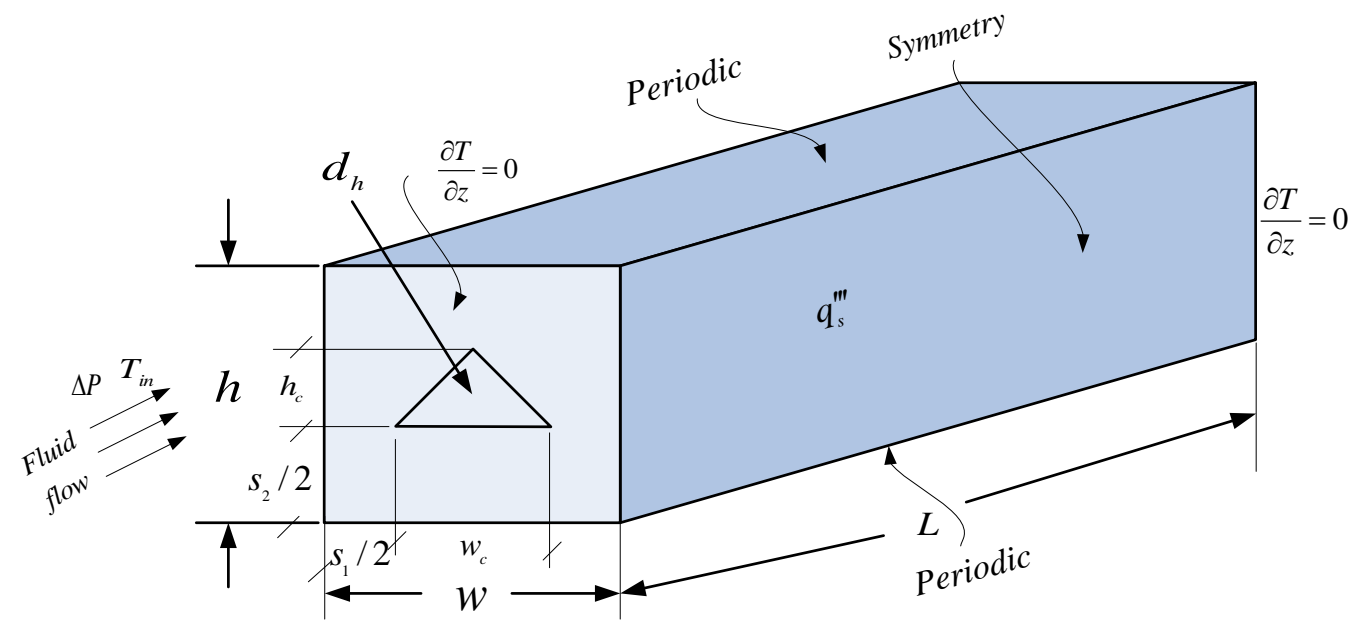

(b)

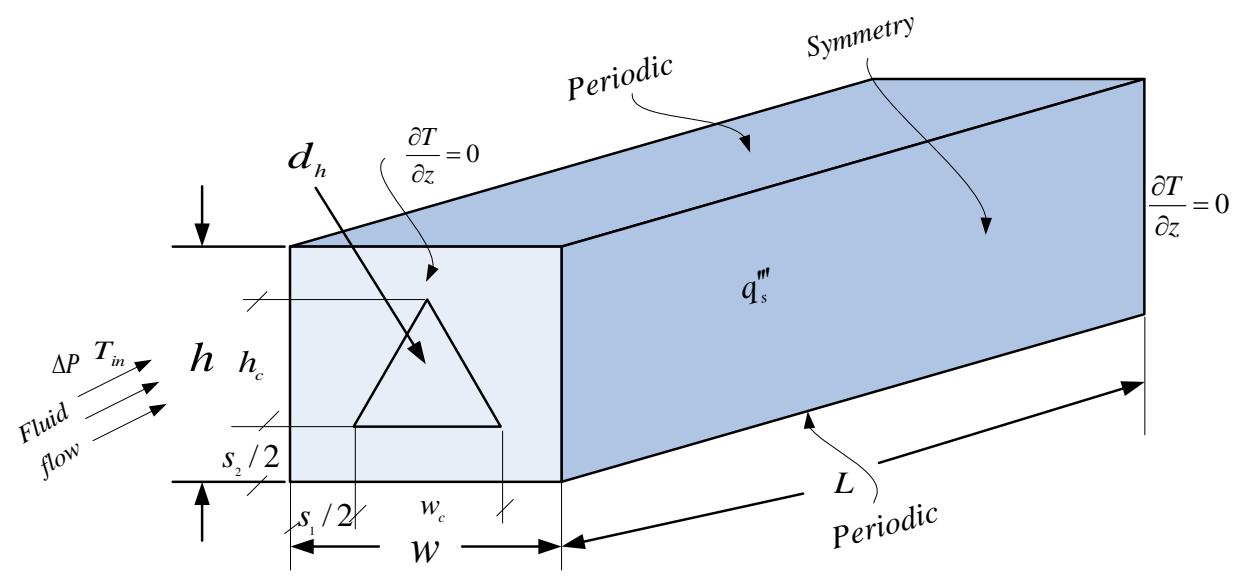

(c)

Figure 1. 


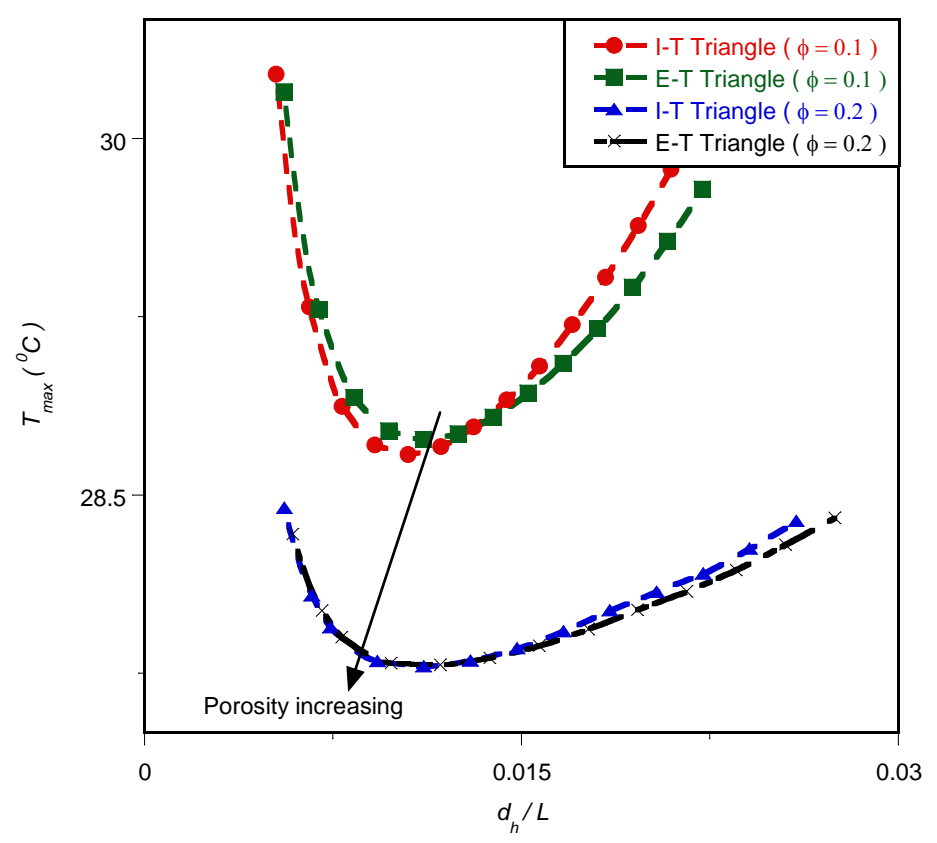

(a)

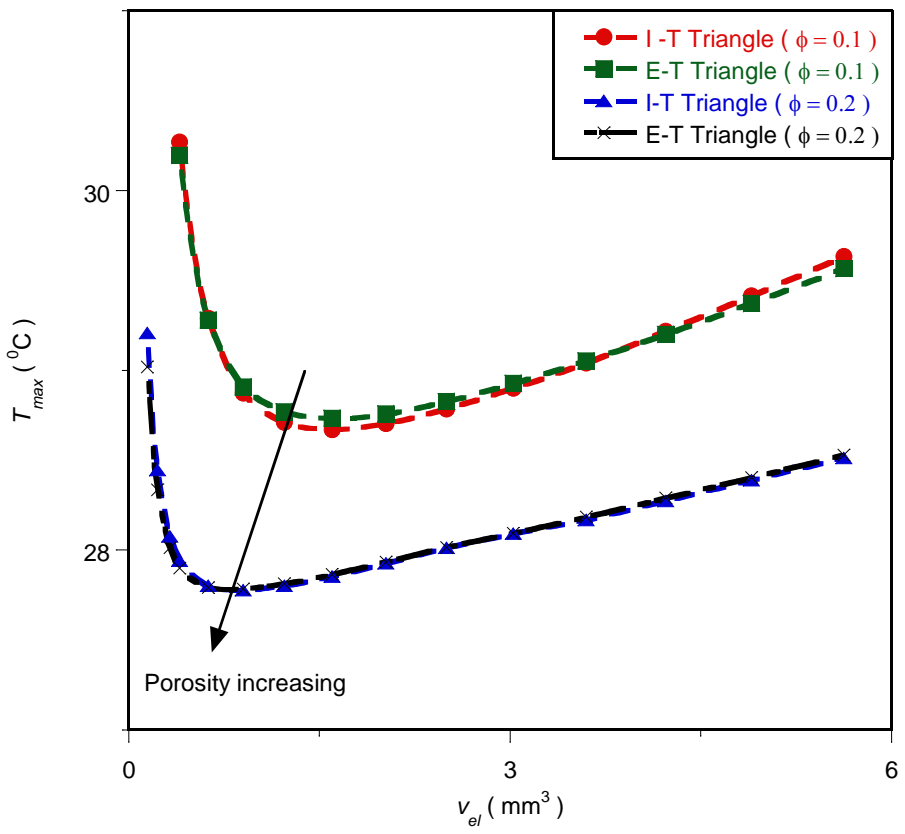

(b)

Figure 2. 


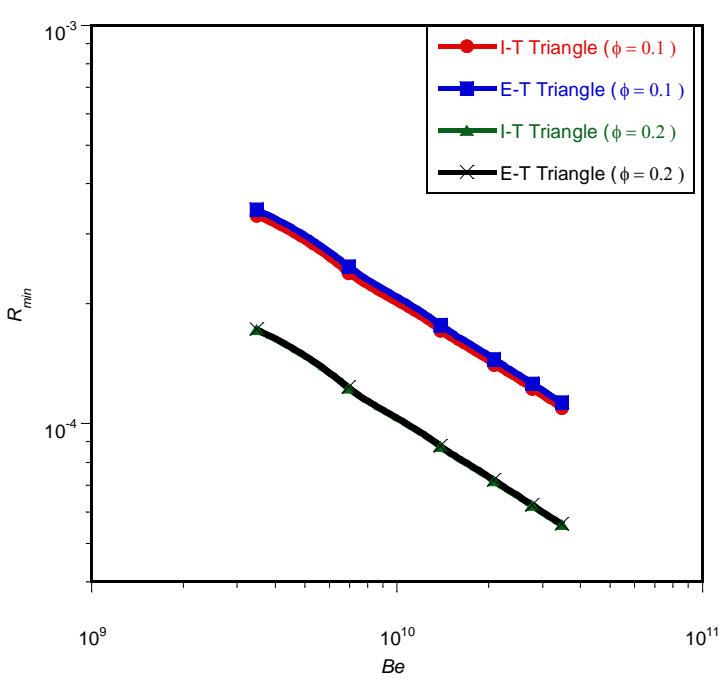

(a)

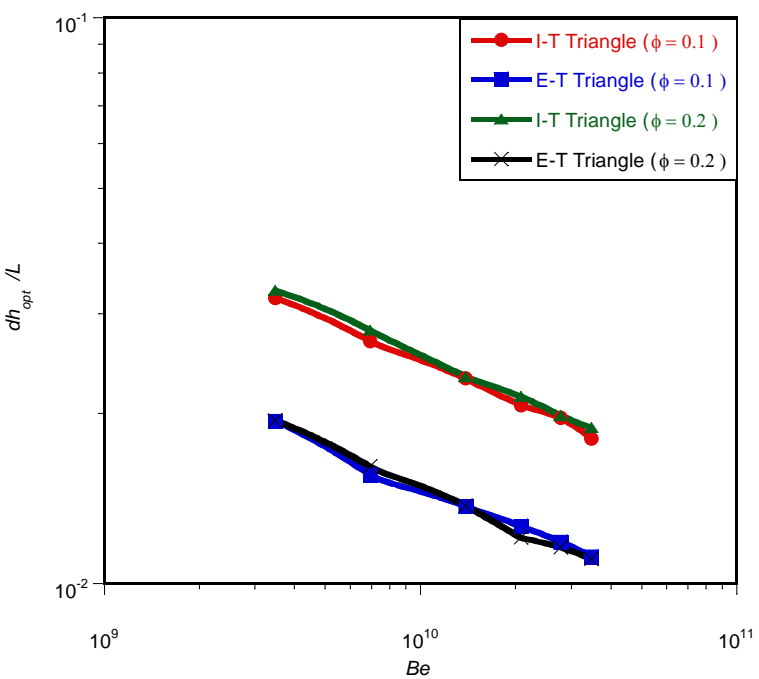

(b)

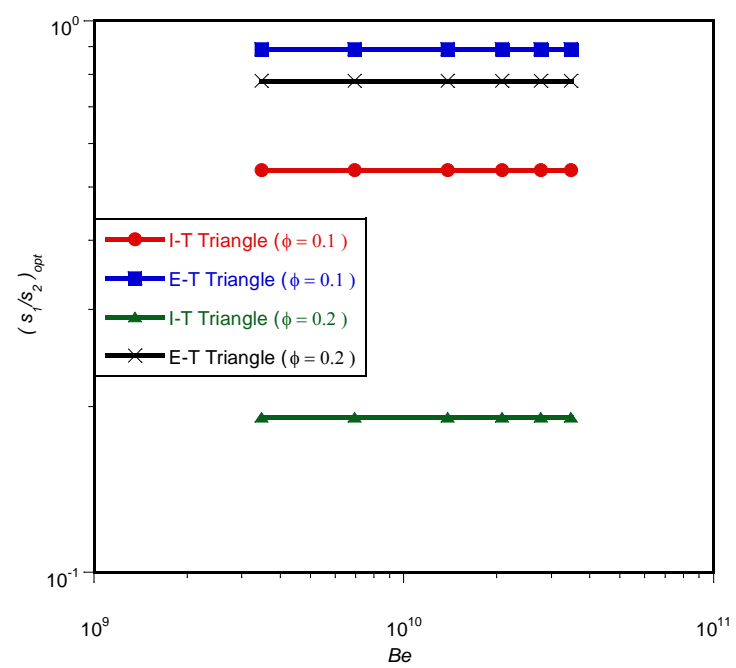

(c)

Figure 3. 


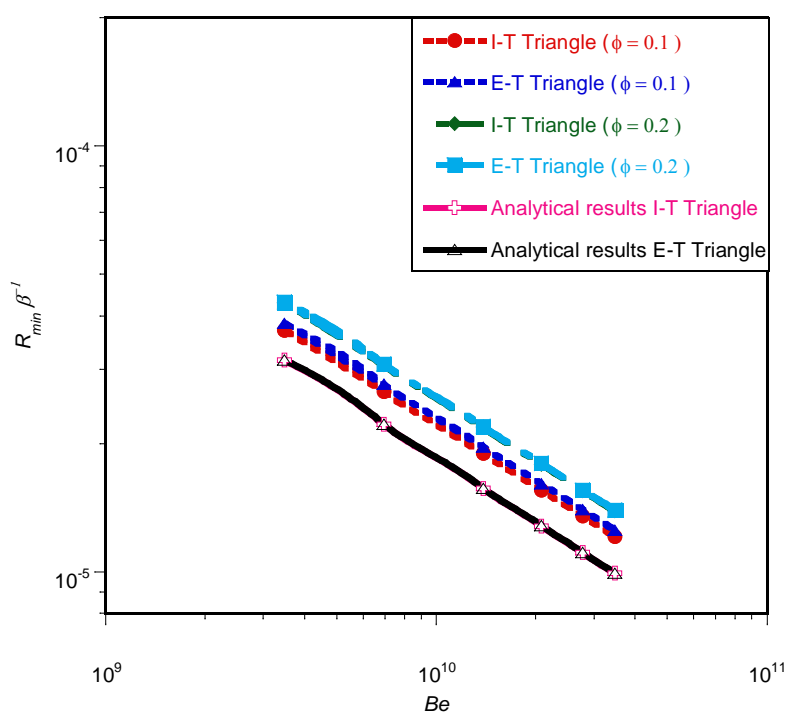

(a)

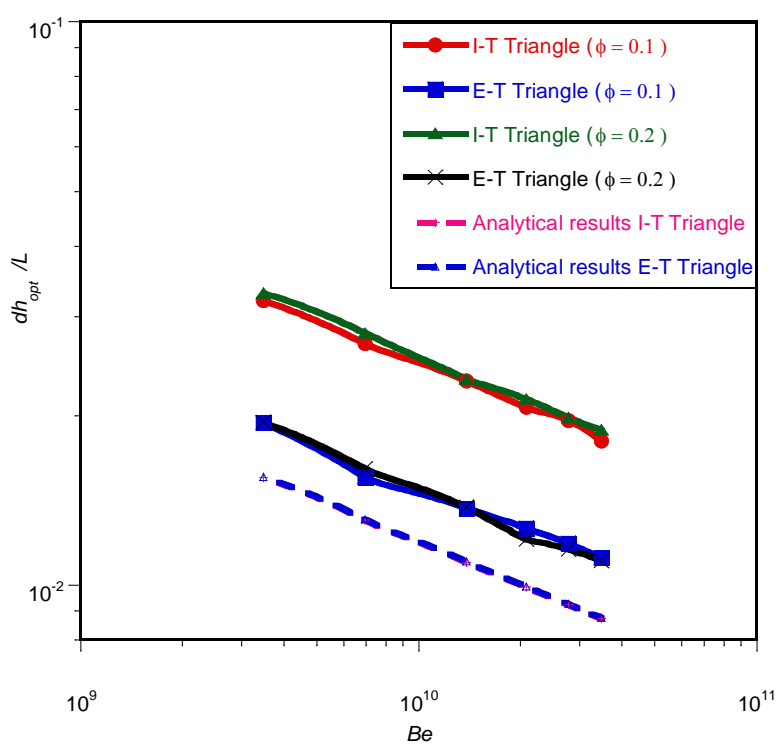

(b)

Figure 4 